\title{
4-1BB agonism: adding the accelerator to cancer immunotherapy
}

\author{
Cariad Chester $^{1,2} \cdot$ Siddhant Ambulkar $^{1} \cdot$ Holbrook E. Kohrt ${ }^{1}$
}

Received: 12 December 2015 / Accepted: 21 March 2016 / Published online: 31 March 2016

(C) The Author(s) 2016. This article is published with open access at Springerlink.com

\begin{abstract}
The success of checkpoint inhibitors has validated immunomodulatory agents as a valuable class of anticancer therapeutics. A promising co-stimulatory immunologic target is 4-1BB, or CD137, a member of the tumor necrosis factor receptor superfamily. Ligation of 4-1BB induces an activating signal in $\mathrm{CD}^{+} \mathrm{T}$ cells and natural killer cells, resulting in increased pro-inflammatory cytokine secretion, cytolytic function, and antibodydependent cell-mediated cytotoxicity. Targeting 4-1BB with agonistic monoclonal antibody (mAb) therapy demonstrated potent antitumor effects in murine tumor models. While anti-4-1BB mAbs have entered clinical trials, optimal efficacy of 4-1BB-targeted agents will inevitably come from combination therapeutic strategies. Checkpoint blockade is a compelling combination partner for $4-1 \mathrm{BB}$
\end{abstract}

It is with great sadness that we note that Holbrook Kohrt passed away on February 24th, 2016.

This paper is a Focussed Research Review based on a presentation given at the Thirteenth Annual Meeting of the Association for Cancer Immunotherapy (CIMT), held in Mainz, Germany, 11th-13th May, 2015. It is part of a series of Focussed Research Reviews and meeting report in Cancer Immunology, Immunotherapy.

Cariad Chester and Siddhant Ambulkar have contributed equally to this work.

\section{Cariad Chester}

cchester@stanford.edu

1 Department of Medicine, Division of Oncology, Stanford University Medical Center, Stanford University, 269 Campus Drive, CCSR 1140, Stanford, CA 94305-5151, USA

2 Institute for Immunity, Transplantation and Infection, Stanford University School of Medicine, Stanford, CA 94305, USA agonism. This novel immunotherapeutic approach has the potential to active antitumor immune effectors by a complementary mechanism: simultaneously "removing the brakes" via blocking inhibitory signaling and "stepping on the accelerator" via co-stimulation. While important considerations should be given to 4-1BB-mediated toxicities, the current understanding of 4-1BB biology suggests it may play a key role in advancing the capabilities of cancer combination therapy.

Keywords CIMT 2015 - 4-1BB · Immunotherapy · Antibody-dependent cell-mediated cytotoxicity . Combination therapy $\cdot$ Checkpoint inhibitors

$\begin{array}{ll}\text { Abbreviations } \\ \text { ADCC } & \text { Antibody-dependent cell-mediated cytotoxicity } \\ \text { ALT } & \text { Alanine aminotransferase } \\ \text { AST } & \text { Aspartate aminotransferase } \\ \text { BMS } & \text { Bristol-Myers Squibb } \\ \text { DLT } & \text { Dose-limiting toxicity } \\ \text { NCT } & \text { National Clinical Trial } \\ \text { NKT } & \text { Natural killer T cell } \\ \text { PF } & \text { Pfizer } \\ \text { TME } & \text { Tumor microenvironment } \\ \text { Treg } & \text { Regulatory T cell }\end{array}$

\section{Introduction}

4-1BB (CD137/TNFRSF9) was discovered in 1989 during screens for novel receptors on murine T-cell lines [1]. Using stimulation with concanavalin A (Con A), Kwon and Weissman identified 4-1BB as an inducible gene on T cells and successfully cloned the 4-1BB cDNA. In the last two decades, our understanding of the biology and function of 
4-1BB has expanded tremendously. 4-1BB is now accepted as an activation-induced, co-stimulatory receptor that governs the function of diverse immune cell subsets. With the advent of cancer immunotherapy, targeting 4-1BB emerged as a promising therapeutic strategy. Analogously to checkpoint inhibitors "removing the brakes" on tumor-targeting effectors, 4-1BB co-stimulation may result in "pressing the accelerator," or increasing immune effector cytotoxicity. Recently, two different monoclonal antibodies (mAb) targeting 4-1BB have entered clinical testing. In this review, we present the current understanding of 4-1BB expression and signaling and discuss the potential of $4-1 \mathrm{BB} \mathrm{mAb}$ therapy to eradicate tumor and synergize with other therapeutic modalities.

\section{4-1BB expression and signaling}

The expression of 4-1BB has been reported on diverse immune cell populations. On T cells, natural killer (NK) cells, regulatory $\mathrm{T}$ cells (Treg), and NK $\mathrm{T}$ cells (NKT), 4-1BB expression is activation dependent [2]. Some of the canonic immunostimulatory agents known to induce 4-1BB upregulation on $\mathrm{T}$ cells include: Con A, plate-bound antiCD3, CD28, phorbol myristoyl acetate (PMA), ionomycin, phytohemagglutinin, interleukin (IL)-2, or IL-4 [3, 4]. On NK cells, 4-1BB is upregulated following ligation of the FcR $\gamma$ III Fc receptor (CD16) by the Fc portion of mAb. In addition to activated immune effectors, 4-1BB is also expressed on innate immune cell populations, including neutrophils, granulocytes, monocytes, mast cells, eosinophils, and dendritic cells (DC) [5]. Expression of 4-1BB has recently been shown on subtypes of lymphomas and leukemias, but its role in malignant transformation remains unclear $[6,7]$.

Once expressed, 4-1BB binds to a high-affinity 4-1BB ligand (4-1BBL or CD137L). 4-1BBL is predominantly expressed by activated antigen-presenting cells (APCs), including B cells, DCs, and macrophages [8]. Upon ligation and crosslinking, 4-1BB associates with the tumor necrosis factor (TNF)-associated factors 1 and 2 (TRAF1 and TRAF2) and activates the master immunoregulatory transcription factor NF- $\kappa \mathrm{B}$. In $\mathrm{T}$ cells, 4-1BB signaling upregulates the antiapoptotic B-cell lymphoma-extra large (Bcl-xl) and B-cell lymphoma 2 (Bcl-2) pathways and induces proliferation and production of pro-inflammatory cytokines interferon gamma (IFN- $\gamma$ ) and IL-2 [9, 10]. Furthermore, 4-1BB stimulation increases signaling through the T-cell receptor (TCR) and amplifies the cytotoxicity of $\mathrm{CD}^{+} \mathrm{T}$ cells [11]. Similarly, in NK cells, CD137 stimulation enhances proliferation, IFN- $\gamma$ production, and cytolytic action [12]. In DCs, CD137 ligation accelerates maturation through upregulation of $\mathrm{B} 7 \mathrm{co}$-stimulatory molecules
(CD80 and CD86) and increases survival and production of IL-6 and IL-12 [13].

\section{4-1BB in cancer immunotherapy}

The initial evidence that anti-4-1BB agonistic mAbs may have strong antitumor effects emerged from studies on the highly tumorigenic P815 mastocytoma and poorly immunogenic Ag104A sarcoma [14]. Administration of anti4-1BB mAb eradicated established tumors in both models and generated increased numbers of tumor-specific cytotoxic $\mathrm{T}$ cells. Importantly, anti-4-1BB mAb therapy appeared to trigger an immunologic memory response: Mice that had been cured of P815 tumor were challenged 3 months later with P815 cells and, after a period of transient growth, all tumors regressed. The antitumor capacity of 4-1BB-targeted $\mathrm{mAb}$ has since been replicated in a variety of tumor models, including lymphoma, hepatocellular carcinoma, and colon cancer [15-17]. The mechanism of action underpinning 4-1BB-mediated tumor regression consists of multiple, complimentary antitumor immune pathways. Primarily, 4-1BB agonism induces a potent, cytotoxic T-cell population that can infiltrate and lyse tumors [18]. In addition to direct tumor lysis, 4-1BB stimulation promotes the secretion of type 1 cytokines, creating an inflammatory, immunogenic cytokine milieu within the tumor microenvironment (TME) [19]. Finally, 4-1BB ligation increases the secretion of perforin and granzyme and activation of the Fas ligand (FasL) effector system by both $\mathrm{CD}^{+} \mathrm{T}$ cells and NK cells [20].

\section{4-1BB stimulation enhanced ADCC}

The role of antibody-dependent cell-mediated cytotoxicity (ADCC) is increasingly appreciated as an important component of antibody-mediated antitumor effects [21, 22]. In ADCC, Fc $\gamma$ RIII (CD16) ligation by the Fc domain of immunoglobulins transmits activating signals within NK cells. In the cancer setting, tumor-targeted $\mathrm{mAb}$ is recognized by NK cell Fc $\gamma$ RIII, triggering release of perforin and granzyme [23]. These direct cytotoxic effects are complemented by a release of interferon gamma (IFN- $\gamma$ ) which can recruit adaptive immune cells and promote antigen presentation by APCs [24]. Human NK cells upregulate 4-1BB after FcRIII ligation, and subsequent stimulation with anti-4-1BB mAb enhances ADCC. Enhanced ADCCmediated tumor clearance makes anti-4-1BB antibodies an ideal candidate for therapeutic combinations with FDA-approved mAbs. We have previously demonstrated that antibodies targeting 4-1BB synergize with rituximab (anti-CD20) and trastuzumab (anti-HER2) to clear tumors 
Table 1 List of anti-4-1BB mAb clinical trials

\begin{tabular}{|c|c|c|c|c|c|}
\hline NCT number ${ }^{a}$ & Phase & Condition & Combination & Start year & Status $(2014 / 12)$ \\
\hline \multicolumn{6}{|c|}{ Urelumab (BMS-663513) $)^{b}$ fully human type IgG4, Bristol-Myers Squibb } \\
\hline NCT00309023 & $\mathrm{I} / \mathrm{II}$ & Metastatic or locally advanced solid tumors & - & 2005 & Terminated \\
\hline NCT00351325 & I & Advanced solid malignancies & Chemotherapy & 2007 & Terminated \\
\hline NCT00461110 & I & Non-small cell lung cancer & Chemoradiation & 2008 & Terminated \\
\hline NCT00612664 & II & Melanoma & - & 2008 & Completed \\
\hline NCT00803374 & I & Advanced malignant melanoma & Ipilimumab (anti-CTLA-4 mAb) & 2010 & Withdrawn \\
\hline NCT01471210 & I & $\begin{array}{l}\text { Advanced and/or metastatic solid tumors } \\
\text { Relapsed/refractory B-cell non-Hodgkin's lymphoma }\end{array}$ & - & 2012 & Recruiting \\
\hline NCT01775631 & $\mathrm{Ib}$ & Relapsed/refractory B-cell non-Hodgkin's lymphoma & Rituximab (anti-CD20 mAb) & 2013 & Recruiting \\
\hline NCT02110082 & $\mathrm{Ib}$ & Colorectal cancer, head and neck cancer & Cetuximab (anti-EGFR mAb) & 2014 & Recruiting \\
\hline NCT02252263 & I & Multiple myeloma & Elotuzumab (anti-CS1 mAb) & 2014 & Recruiting \\
\hline \multicolumn{5}{|c|}{ PF-05082566 : fully human type IgG2, Pfizer } & Recruiting \\
\hline NCT01307267 & I & CD20 positive non-Hodgkin's lymphoma & Rituximab (anti-CD20 mAb) & 2011 & Recruiting \\
\hline NCT02179918 & $\mathrm{Ib}$ & Advanced solid tumors & MK-3475 (anti-PD-1 mAb) & 2014 & Recruiting \\
\hline
\end{tabular}

in murine xenograft models of lymphoma and breast cancer $[25,26]$. The synergistic therapeutic effect was dependent on Fc $\gamma$ RIII expression and observed in mice competent in NK cells and macrophages, but lacking an adaptive immune system. Recently, we combined cetuximab (antiEGFR) and anti-4-1BB antibody therapy to obtain complete tumor resolution and prolonged survival in models of colorectal cancer and head and neck cancer [27]. Based on the preclinical successes of antibodies targeting 4-1BB, two agonistic anti-4-1BB mAb entered clinical testing.

\section{4-1BB clinical trials}

The two anti-4-1BB mAbs currently in the clinic are urelumab (Bristol-Myers Squibb), a fully humanized IgG4 $\mathrm{mAb}$, and PF-05082566 (Pfizer), a fully human IgG2 mAb (Table 1). Urelumab is now being tested in multiple combinatorial regimens: cetuximab in head and neck or colorectal cancer, rituximab in relapsed or refractory B-cell NHL, and elotuzumab (anti-SLAMF7) in multiple myeloma. PF-05082566 is currently in three clinical trials, and results of the phase I monotherapy study were recently reported [28]. Out of 27 treated patients, the best clinical response was disease stabilization, occurring in $22 \%$ (6/27) of patients. Importantly, PF-05082566 did not induce any dose-limiting toxicities (DLTs), and all study discontinuations were from disease progression, not adverse events. Ongoing clinical studies test PF-05082566 in combination with rituximab and mogamulizumab (anti-CCR4).
As anti-4-1BB targeted agents advance in clinical trials, monitoring potential toxicities is a chief responsibility. Liver inflammation was observed during 4-1BB monotherapy, characterized by elevated levels of aspartate aminotransferase (AST) and alanine aminotransferase (ALT). Grade 2+ neutropenia, leukopenia, and thrombocytopenia were also observed [29]. Potentially fatal hepatitis was later observed in a Bristol-Myers Squibb (BMS) phase II anti-4-1BB study for previously treated stage III/IV melanoma, National Clinical Trial (NCT) 00612664. This study and several others (NCT00803374, NCT00309023, NCT00461110, NCT00351325) were terminated due to these adverse events. Despite this clinical hurdle, further studies have proven that hepatic toxicity due to 4-1BB agonist antibodies can be largely mitigated by lowering the dose of anti-4-1BB mAb administered.

\section{4-1BB and checkpoint inhibitors}

Checkpoint inhibitors are a novel and promising class of immunotherapeutic antibodies and have evoked impressive clinical responses to melanoma, non-small-cell lung cancer, and renal cancer. Immune checkpoints are inhibitory pathways that downregulate activated $\mathrm{T}$ cells following antigen presentation and co-stimulatory signaling by APCs. Currently, two checkpoint receptors have been targeted by FDA-approved mAbs: CTLA-4, targeted by ipilimumab, and programmed death (PD)-1. The recent approvals of PD-1 blocking mAb pembrolizumab and nivolumab 
validate the effectiveness of checkpoint blockade strategy in the setting of clinical oncology. Conceptually, the addition of 4-1BB agonists to a therapeutic backbone of checkpoint blockade is highly appealing. This combination targets two distinct elements in the process of immune effector activation: overcoming inhibition and co-stimulation. Checkpoint inhibitors can block the inhibitory signals that pervade the immunosuppressive TME, while 4-1BB stimulation can augment effector cells' baseline cytotoxic capacity. If both molecules are simultaneously targeted, selective activation of tumor-targeting $\mathrm{T}$ and NK cells may occur, releasing a robust antitumor immune response. To date, there is strong clinical preclinical evidence to support this combination.

The combination of anti-CTLA-4 antibodies and anti4-1BB has demonstrated preclinical success in multiple tumor histologies. In the B16 melanoma model, the combination showed a synergistic curative effect and induced antitumor TME remodeling; after therapy, the number of $\mathrm{CD}^{+} \mathrm{T}$ cells infiltrating the tumor increased, while the numbers of Tregs and myeloid-derived suppressor cells (MDSCs) decreased [30]. In the RM-1 prostate cancer model, a 4-1BBL-expressing tumor cell vaccine combined with CTLA-4 blockade caused tumor rejection in four out of five mice implanted with RM-1 tumors [31]. In the MC38 colon adenocarcinoma model, the combination of anti-4-1BB mAb and anti-CTLA-4 antibody led to the eradiation of large, established tumors $(>7 \mathrm{~mm}$ in diameter) in seven out of eight mice and induced long-lasting immunity to rechallenge with MC38 cells [32]. More importantly than the observed synergistic therapeutic activity, Kocak et al. [32] demonstrated that combining anti-4-1BB with anti-CTLA-4 has the potential to ameliorate the toxicities observed with monotherapeutic use of anti-4-1BB mAb. Anti-CTLA-4 therapy reduced the 4-1BB-induced influx of $T$ cell infiltrates into the liver and prevented the hepatic toxicity that has limited 4-1BB application in the clinic.

The PD-1 blocking mAbs nivolumab and pembrolizumab have demonstrated comparable therapeutic activity to ipilimumab while also having a milder toxicity profile. These observations suggest that PD-1 blockade may be superior to anti-CTLA-4 antibodies for combination with 4-1BB agonists. In one of the first preclinical models of this therapeutic strategy, Xiao et al. [33] combined soluble PD-1 with naked plasmid p4-1BBL-transfected H22 tumor cells in an ectopic hepatocarcinoma model. This combination demonstrated robust therapeutic synergy, increasing the number of infiltrating $\mathrm{T}$ cells and eradicating $60 \%$ of established tumors. Recent testing of anti-4-1BB with PD-1 blockade further supports the argument for combinatorial synergy. In the CT26 colon adenocarcinoma model, combining 4-1BB agonism and anti-PD-1 mAb resulted in complete tumor rejection in all mice injected with CT26 cells [34]. In the poorly immunogenic B16-F10 melanoma model, combination therapy again resulted in complete tumor regression and an increased ratio of $\mathrm{CD}^{+} \mathrm{T}$ cells to Tregs [35]. However, Chen et al. [35] also demonstrated that the combination of anti-4-1BB and anti-PD-1 may exacerbate toxicities observed with anti-4-1BB monotherapy. At the higher dose levels of anti-4-1BB mAb (1 and $5 \mathrm{mg} / \mathrm{kg}$ ), AST and ALT levels were elevated over twofold what was reported with anti-4-1BB monotherapy and the combination intensified anti-4-1BB-induced neutropenia, lymphopenia, and thrombocytopenia. These results suggest that PD-1 blockade may be a potent therapeutic partner for anti-4-1BB, but clinicians must progress cautiously with early-phase trials until an appropriate dosage is established.

Another approach to inhibiting the PD-1-mediated suppressive signaling is to block the ligand of PD-1 ligand (PD-L1). This approach has exhibited exciting early data, and currently, four clinical anti-PD-L1 mAbs are in testing: atezolizumab and MEDI4736 (both Fc null variants of human IgG1), MSB001078C (IgG1), and BMS-936559 (IgG4). In the ID-8 ovarian adenocarcinoma model, the combination of anti-PD-L1 and anti-4-1BB antibodies increased overall survival and enhanced T-cell effector function [36]. As anti-PD-L1 mAbs advance in clinical trials, combination therapy with anti-4-1BB mAbs will undoubtedly receive increased consideration. Currently, 4-1BB and anti-PD-1 checkpoint inhibitors are the focus of ongoing clinical trials. A phase I/II trial of urelumab with nivolumab in both solid tumors and B-cell non-Hodgkin's lymphoma is recruiting participants (NCT02253992), while PF-05082566 is being tested in a phase Ib trial with pembrolizumab in patients with solid tumors (NCT02179918).

\section{4-1BB and cancer vaccines}

Recently, there has been a renewed focus on therapeutic cancer vaccines. The allure of vaccine strategies is the potential to "teach" individual patients' immune systems to recognize, target, and eradicate tumor cells in an approach that employs both adaptive and innate immunity. Vaccines aim to provoke a tumor-specific immune response by increasing TAA presentation by APCs, thus generating tumor antigen-specific cytotoxic $\mathrm{T}$ lymphocytes. Unlike chemotherapy, surgery, radiotherapy, or antibody therapy, an effective vaccine-induced immune response could establish a state of immunologic memory that persists after tumor clearance and indefinitely suppresses tumor regrowth. Mechanistically, the combination of vaccine strategies and anti-4-1BB targeted agents is compelling because it targets synergistic and non-overlapping components of the antitumor immune response; vaccines generate an immune response and anti-4-1BB further activates tumor-targeted effector populations. 
Ongoing efforts to combine cancer vaccines and anti4-1BB agents have demonstrated early progress. In the HPV E6/E7-driven TC-1 murine cervical cancer model, vaccination with HPV E6/E7 peptides in conjunction with systemic 4-1BB agonistic antibody induced durable tumor regression in five out of eight mice. Surviving mice that were rechallenged with additional TC- 1 cells demonstrated strong $\mathrm{CD}^{+} \mathrm{T}$ cell memory responses, remaining tumor free. Anti-4-1BB antibodies proved to be a superior combination partner for vaccine therapy, demonstrating superior results relative to CTLA-4 and PD- 1 checkpoint inhibitors as well as other TNF family receptors [37]. In a MCA205 murine fibrosarcoma model, a tumor lysate-pulsed dendritic cell vaccine (TP-DC) combined with $\alpha 4-1 B B$ significantly reduced lung metastases. The combined therapy also led to regression of lung tumors in a B16-BL6 melanoma model [38].

\section{Conclusion}

Significant progress has been made in recent years in elucidating the expression, function, and therapeutic potential of 4-1BB. The ongoing clinical trials dedicated to 4-1BB agonists portend the viability of $4-1 \mathrm{BB}$ as an important immunotherapeutic target. The maximal clinical efficacy of 4-1BB will emerge in combination treatment strategies, and checkpoint inhibitors are a compelling class of partner candidates. However, the broad range of 4-1BB expression makes off-target immune pathology an acute risk. Further research is needed to optimize approaches to harnessing 4-1BB-mediated antitumor effects, while minimizing autoinflammatory complications. If this goal can be achieved, 4-1BB agonists may provide a critical component of future anticancer combination therapies.

\section{Compliance with ethical standards}

Conflict of interest The authors declared that no conflicts of interest exist.

Open Access This article is distributed under the terms of the Creative Commons Attribution 4.0 International License (http://creativecommons.org/licenses/by/4.0/), which permits unrestricted use, distribution, and reproduction in any medium, provided you give appropriate credit to the original author(s) and the source, provide a link to the Creative Commons license, and indicate if changes were made.

\section{References}

1. Kwon BS, Weissman SM (1989) cDNA sequences of two inducible T-cell genes. Proc Natl Acad Sci USA 86:1963-1967
2. Schoenbrunn A, Frentsch M, Kohler S, Keye J et al (2012) A converse 4-1BB and CD40 ligand expression pattern delineates activated regulatory $\mathrm{T}$ cells (Treg) and conventional $\mathrm{T}$ cells enabling direct isolation of alloantigen-reactive natural Foxp3+ Treg. J Immunol 189:5985-5994

3. Watts TH (2005) TNF/TNFR family members in costimulation of T cell responses. Annu Rev Immunol 23:23-68

4. Vinay DS, Kwon BS (1998) Role of 4-1BB in immune responses. Semin Immunol 10:481-489

5. Vinay DS, Kwon BS (2011) 4-1BB signaling beyond T cells. Cell Mol Immunol 8:281-284

6. Anderson MW, Zhao S, Freud AG, Czerwinski DK et al (2012) CD137 is expressed in follicular dendritic cell tumors and in classical Hodgkin and T-cell lymphomas: diagnostic and therapeutic implications. Am J Pathol 181:795-803

7. Pang WL, Ho WT, Schwarz H (2013) Ectopic CD137 expression facilitates the escape of Hodgkin and Reed-Sternberg cells from immunosurveillance. Oncoimmunology 2:e23441

8. Wang C, Lin GHY, McPherson AJ, Watts TH (2009) Immune regulation by 4-1BB and 4-1BBL: complexities and challenges. Immunol Rev 229:192-215

9. Lee HW, Park SJ, Choi BK, Kim HH et al (2002) 4-1BB promotes the survival of $\mathrm{CD} 8^{+} \mathrm{T}$ lymphocytes by increasing expression of Bcl-xL and Bfl-1. J Immunol 169:4882-4888

10. Snell LM, Lin GH, McPherson AJ, Moraes TJ, Watts TH (2011) T-cell intrinsic effects of GITR and 4-1BB during viral infection and cancer immunotherapy. Immunol Rev 244:197-217

11. Shuford WW, Klussman K, Tritchler DD, Loo DT et al (1997) 4-1BB costimulatory signals preferentially induce CD8 + T cell proliferation and lead to the amplification in vivo of cytotoxic $\mathrm{T}$ cell responses. J Exp Med 186:47-55

12. Melero I, Johnston JV, Shufford WW, Mittler RS, Chen L (1998) NK1.1 cells express 4-1BB (CDw137) costimulatory molecule and are required for tumor immunity elicited by anti-4-1BB monoclonal antibodies. Cell Immunol 190:167-172

13. Kuang Y, Weng X, Liu X, Zhu H et al (2012) Effects of 4-1BB signaling on the biological function of murine dendritic cells. Oncol Lett 3:477-481

14. Melero I, Shuford WW, Newby SA, Aruffo A et al (1997) Monoclonal antibodies against the 4-1BB T-cell activation molecule eradicate established tumors. Nat Med 3:682-685

15. Houot R, Goldstein MJ, Kohrt HE, Myklebust JH et al (2009) Therapeutic effect of CD137 immunomodulation in lymphoma and its enhancement by Treg depletion. Blood 114:3431-3438

16. Gauttier V, Judor JP, Le Guen V, Cany J et al (2014) Agonistic anti-CD137 antibody treatment leads to antitumor response in mice with liver cancer. Int J Cancer 135:2857-2867

17. Li B, Lin J, Vanroey M, Jure-Kunkel M, Jooss K (2007) Established B16 tumors are rejected following treatment with GMCSF-secreting tumor cell immunotherapy in combination with anti-4-1BB mAb. Clin Immunol 125:76-87

18. Curran MA, Geiger TL, Montalvo W, Kim M et al (2013) Systemic 4-1BB activation induces a novel $\mathrm{T}$ cell phenotype driven by high expression of Eomesodermin. J Exp Med 210:743-755

19. Li Q, Carr A, Ito F, Teitz-Tennenbaum S, Chang AE (2003) Polarization effects of 4-1BB during CD28 costimulation in generating tumor-reactive $\mathrm{T}$ cells for cancer immunotherapy. Cancer Res 63:2546-2552

20. Morales-Kastresana A, Catalán E, Hervás-Stubbs S, Palazón A et al (2013) Essential complicity of perforin-granzyme and FAS-L mechanisms to achieve tumor rejection following treatment with anti-CD137 mAb. J Immunother Cancer 1:3

21. Chester C, Marabelle A, Houot R, Kohrt HE (2015) Dual antibody therapy to harness the innate anti-tumor immune response to enhance antibody targeting of tumors. Curr Opin Immunol 33:1-8 
22. Offringa R, Glennie MJ (2015) Development of next-generation immunomodulatory antibodies for cancer therapy through optimization of the IgG framework. Cancer Cell 28:273-275

23. Wang W, Erbe AK, Hank JA, Morris ZS, Sondel PM (2015) NK Cell-mediated antibody-dependent cellular cytotoxicity in cancer immunotherapy. Front Immunol 6:368

24. Srivastava RM, Lee SC, Andrade Filho PA, Lord CA et al (2013) Cetuximab-activated natural killer and dendritic cells collaborate to trigger tumor antigen-specific T-cell immunity in head and neck cancer patients. Clin Cancer Res 19:1858-1872

25. Kohrt HE, Houot R, Goldstein MJ, Weiskopf K et al (2011) CD137 stimulation enhances the antilymphoma activity of antiCD20 antibodies. Blood 117:2423-2432

26. Kohrt HE, Houot R, Weiskopf K, Goldstein MJ et al (2012) Stimulation of natural killer cells with a CD137-specific antibody enhances trastuzumab efficacy in xenotransplant models of breast cancer. J Clin Invest 122:1066-1075

27. Kohrt HE, Colevas AD, Houot R, Weiskopf K et al (2014) Targeting CD137 enhances the efficacy of cetuximab. J Clin Invest 124:2668-2682

28. Segal NH, Gopal AK, Bhatia S, Kohrt HE, Levy R et al (2014) A phase 1 study of PF-05082566 (anti-4-1BB) in patients with advanced cancer. J Clin Oncol 32:5 (suppl; abstr 3007)

29. Ascierto PA, Simeone E, Sznol M, Fu YX, Melero I (2010) Clinical experiences with anti-CD137 and anti-PD1 therapeutic antibodies. Semin Oncol 37(5):508-516

30. Curran MA, Kim M, Montalvo W, Al-Shamkhani A, Allison JP (2011) Combination CTLA-4 blockade and 4-1BB activation enhances tumor rejection by increasing T-cell infiltration, proliferation, and cytokine production. PLoS One 6:e19499
31. Youlin K, Li Z, Xiaodong W, Xiuheng L, Hengchen Z (2012) Combination immunotherapy with 4-1BBL and CTLA-4 blockade for the treatment of prostate cancer. Clin Dev Immunol 2012:439235

32. Kocak E, Lute K, Chang X, May KF Jr et al (2006) Combination therapy with anti-CTL antigen-4 and anti-4-1BB antibodies enhances cancer immunity and reduces autoimmunity. Cancer Res 66:7276-7284

33. Xiao H, Huang B, Yuan Y, Li D et al (2007) Soluble PD-1 facilitates 4-1BBL-triggered antitumor immunity against murine $\mathrm{H} 22$ hepatocarcinoma in vivo. Clin Cancer Res 13:1823-1830

34. Shindo Y, Yoshimura K, Kuramasu A, Watanabe Y et al (2015) Combination immunotherapy with 4-1BB activation and PD-1 blockade enhances antitumor efficacy in a mouse model of subcutaneous tumor. Anticancer Res 35:129-136

35. Chen F, Fan C, Gu X, Zhang H et al (2015) Construction of antiCD20 single-chain antibody-CD28-CD137-TCRל recombinant genetic modified $\mathrm{T}$ cells and its treatment effect on B cell lymphoma. Med Sci Monit 21:2110-2115

36. Duraiswamy J, Freeman GJ, Coukos G (2013) Therapeutic PD-1 pathway blockade augments with other modalities of immunotherapy T-cell function to prevent immune decline in ovarian cancer. Cancer Res 73:6900-6912

37. Bartkowiak T, Curran MA (2015) 4-1BB agonists: multi-potent potentiators of tumor immunity. Front Oncol 5:117

38. Ito F, Li Q, Shreiner AB, Okuyama R et al (2004) Anti-CD137 monoclonal antibody administration augments the antitumor efficacy of dendritic cell-based vaccines. Cancer Res 64:8411-8419 